\title{
Polyclonal Intestinal Colonization with Extended-Spectrum Cephalosporin-Resistant Enterobacteriaceae upon Traveling to India
}

\author{
João Pires ${ }^{1,2}$, Esther Kuenzli ${ }^{3,4,5}$, Sara Kasraian ${ }^{1}$, Regula Tinguely ${ }^{1}$, Hansjakob Furrer ${ }^{6}$, \\ Markus Hilty ${ }^{1,6}$, Christoph Hatz $^{4,5}$ and Andrea Endimiani ${ }^{1 *}$ \\ ${ }^{1}$ Institute for Infectious Diseases, University of Bern, Bern, Switzerland, ${ }^{2}$ Graduate School of Cellular and Biomedical \\ Sciences, University of Bern, Bern, Switzerland, ${ }^{3}$ Division for Infectious Diseases and Hospital Epidemiology, University \\ Hospital Basel, Basel, Switzerland, ${ }^{4}$ Swiss Tropical and Public Health Institute, Basel, Switzerland, ${ }^{5}$ Epidemiology, \\ Biostatistics and Prevention Institute, University of Zurich, Zurich, Switzerland, ${ }^{6}$ Department of Infectious Diseases, Bern \\ University Hospital, University of Bern, Bern, Switzerland
}

OPEN ACCESS

Edited by:

Paolo Visca,

Roma Tre University, Italy

Reviewed by:

Sebastian Guenther,

Freie Universität Berlin, Germany

Jorge Blanco,

University of Santiago de Compostela,

Spain

*Correspondence:

Andrea Endimiani

andrea.endimiani@ifik.unibe.ch

aendimiani@gmail.com

Specialty section:

This article was submitted to

Antimicrobials, Resistance and

Chemotherapy,

a section of the journal

Frontiers in Microbiology

Received: 30 May 2016

Accepted: 27 June 2016

Published: 12 July 2016

Citation:

Pires J, Kuenzli E, Kasraian S, Tinguely $R$, Furrer $\mathrm{H}$, Hilty M, Hatz C and Endimiani A (2016) Polyclonal Intestinal Colonization with

Extended-Spectrum

Cephalosporin-Resistant Enterobacteriaceae upon Traveling to India. Front. Microbiol. 7:1069. doi: 10.3389/fmicb.2016.01069
We aimed to assess the intestinal colonization dynamics by multiple extended-spectrum cephalosporin-resistant Enterobacteriaceae (ESC-R-Ent) clones in Swiss travelers to India, a country with high prevalence of these multidrug-resistant pathogens. Fifteen healthy volunteers (HVs) colonized with ESC-R-Ent after traveling to India who provided stools before, after, and at 3- and 6-month follow-up are presented in this study. Stools were enriched in a LB broth containing $3 \mathrm{mg} / \mathrm{L}$ cefuroxime and plated in standard selective media (BLSE, ChromID ESBL, Supercarba) to detect carbapenem- and/or ESC-R-Ent. At least 5 Enterobacteriaceae colonies were analyzed for each stool provided. All strains underwent phenotypic tests (MICs in microdilution) and molecular typing to define bla genes (microarray, PCR/sequencing), clonality (MLST, rep-PCR), and plasmid content. While only three HVs were colonized before the trip, all participants had positive stools after returning, but the colonization rate decreased during the follow-up period (i.e., six HVs were still colonized at both 3 and 6 months). More importantly, polyclonal acquisition (median of 2 clones, range 1-5) was identified at return in all HVs. The majority of the Escherichia coli isolates belonged to phylogenetic groups A and B1 and to high diverse non-epidemic sequence types (STs); however, 15\% of them belonged to clonal complex 10 and mainly possessed bla $\mathrm{CTX}-\mathrm{M}-15$ genes. $\mathrm{F}$ family plasmids were constantly found $(\sim 80 \%)$ in the recovered ESC-R-Ent. Our results indicate a possible polyclonal acquisition of the ESC-R-Ent via food-chain and/or through an environmental exposure. For some HVs, prolonged colonization in the follow-up period was observed due to clonal persistence or presence of the same plasmid replicon types in a new bacterial host. Travel medicine practitioners, clinicians, and clinical microbiologists who are facing the returning travelers and their samples for different reasons should be aware of this important phenomenon, so that better infection control measures, treatment strategies, and diagnostic tests can be adopted.

Keywords: E. coli, travelers, food-chain, environment, pAmpC, ESBL, CTX-M, plasmid 


\section{INTRODUCTION}

The human gut is increasingly recognized as an important reservoir of extended-spectrum cephalosporin-resistant Enterobacteriaceae (ESC-R-Ent), as increasing colonization rates by these pathogens are observed worldwide (Woerther et al., 2013). In particular, being colonized by ESBL-producing Enterobacteriaceae (ESBL-Ent) constitutes a risk factor to develop future extra-intestinal infections. Despite this fact, silent carriage of these multidrug-resistant (MDR) bacteria in the human gut is still poorly studied (Rogers et al., 2011; Van Der Bij and Pitout, 2012).

The highest colonization prevalence in the healthy population ( $\sim 70 \%$ ) has been reported in Southeast Asia (Woerther et al., 2013). Rates increased exponentially over the last decade and can be linked to several main factors: importation of highrisk clones (HiRC) via intercontinental travel, inappropriate use of antibiotics in different settings, high resistance rates in hospitals, poor hygienic measures (including food-handling), and high population density (Woerther et al., 2013). Therefore, it is not surprising that traveling to high prevalence countries constitute a risk factor to become colonized with these organisms (Van Der Bij and Pitout, 2012). Moreover, this importation of bacteria might facilitate the spread of MDR clones in low prevalence regions (Memish et al., 2003; Rogers et al., 2011). The problem has been highlighted in previous studies which reported individuals who acquired infections abroad with organisms encoding for new life-threatening resistance mechanisms (e.g., the first NDM-1 producer in a patient returning to Sweden or the importation of colistin-resistant MCR-1 producers from India), or which described resistance mechanisms in new bacterial hosts and/or genetic structures (Yong et al., 2009; Rogers et al., 2011; Seiffert et al., 2014; Bernasconi et al., 2016).

In recent years, an increased awareness for polymicrobial and/or polybacterial infections has also been achieved due to increased sensitivity of the microbiology diagnostic procedures (Short et al., 2014; Endimiani and Jacobs, 2016). However, we note that very few studies have identified multiple ESC-R-Ent colonizing the gut of healthy individuals (especially returning travelers) (Paltansing et al., 2013; Girlich et al., 2015; Stoesser et al., 2015), and very little attention has been attributed to this phenomenon.

In this work, we aimed to assess the colonization dynamics by multiple ESC-R-Ent clones acquired during a travel to India, a high prevalence country of ESC-R-Ent, to further understand the influence of this phenomenon in the shaping of the population structure of ESC-R-Ent in the human gut.

\section{MATERIALS AND METHODS}

\section{Recruitment of Volunteers and Study Design}

Fifteen healthy volunteers (HVs) traveling to India were selected from a larger ongoing study to perform this longitudinal pilot analysis. These HVs were chosen because they: (i) provided stool samples before and after traveling (both within 1 week), (ii) were colonized with ESC-R-Ent after returning from their trip, and (iii) provided follow-up samples at 3 and 6 months after the trip.

All HVs were recruited at the travel clinic of the Swiss Tropical and Public Health Institute (Basel) where they signed an informed consent. Subjects were instructed to self-collect the native stools in sterile plastic containers and send them to the Institute for Infectious Diseases of the University of Bern (Switzerland). Ethical approval was obtained by the Ethikkommission Nordwest- und Zentralschweiz (EKNZ 239/12).

\section{Sample Processing and Isolate Recovery}

Stools $(20 \mu \mathrm{g})$ were enriched overnight in $10 \mathrm{~mL}$ of Luria-Bertani (LB) broth containing a $30 \mu \mathrm{g}$ cefuroxime disc. Enrichments were plated $(50 \mu \mathrm{L})$ in BLSE, ChromID ESBL (bioMérieux), and a modified Supercarba plate containing imipenem to detect carbapenem- and/or ESC-R-Ent (Viau et al., 2016).

Species identification was obtained by using the MALDI-TOF MS (Bruker). At least 5 Enterobacteriaceae colonies per stool sample were analyzed. Strains were submitted to the double-disk synergism test (DDST) in cation-adjusted Mueller-Hinton plates (Becton Dickinson) supplemented with and without cloxacillin (250 mg/L; Sigma-Aldrich). Isolates yielding DDST results compatible with ESBL or plasmid-mediated AmpC (pAmpC) production were further characterized.

\section{Antimicrobial Susceptibility Testing (AST) and $\beta$-lactamase(s) Characterization}

ASTs were obtained using the microdilution Sentitre ${ }^{\mathrm{TM}}$ GNX2F plate (Trek Diagnostic Systems) and interpreted with EUCAST breakpoints, except for doxycycline and minocycline for which CLSI guidelines were used (CLSI, 2016; Eucast, 2016).

$\beta$-lactamase genes (bla) were identified using the CT103XL microarray (Check-Points). PCR/sequencing of the bla $a_{\mathrm{ESBL}}$ and $b l a_{\text {pAmpC }}$ genes was carried out as previously done (Seiffert et al., 2013).

\section{Analysis of Clonality and Plasmid Content}

The repetitive extragenic palindromic PCR (rep-PCR) was implemented to assess clonal relatedness (Seiffert et al., 2013). Multilocus Sequence Typing (MLST) for E. coli (http://mlst. ucc.ie/mlst/dbs/Ecoli) and K. pneumoniae (http://bigsdb.web. pasteur.fr/klebsiella/klebsiella.html) were also performed. The phylogenetic group (PhG) for E. coli was also obtained (EscobarPáramo et al., 2004).

Plasmids' incompatibility groups were assessed with the multiplex PCR based replicon typing (PBRT) kit (Diatheva).

\section{RESULTS}

\section{Demographic and Clinical Characteristics of Travelers}

As shown in Table 1, HVs were mostly females (73.3\%) and middle-aged adults (average of 44 years). None of them was vegetarian or took anti-acids regularly. The average length of stay in India was 17.6 days (range 9-30 days). Four subjects reported having suffered from travelers' diarrhea. None of the HVs took 
antibiotics during their trip. During the 6-month follow-up period, several HVs traveled to other destinations (including India again).

\section{Colonization Timeline and Individual ESC-R-Ent Clones}

Only three out of 15 HVs were colonized by ESC-R-Ent before traveling, whereas all of them had positive stools after return (Table 2). During the follow up period, six subjects were colonized at both 3 and 6 months.

Among the $15 \mathrm{HVs}$, a total of 143 isolates yielded DDST phenotypes compatible with either ESBL or pAmpC production. rep-PCR analysis within each time point narrowed down the number of ESC-R-Ent isolates to 46 unique clones (data not shown), consisting of $44 \mathrm{E}$. coli, one K. pneumoniae, and one Proteus mirabilis. MLST analysis was performed in this subgroup of strains. Notably, ESC-R species other than E. coli were only recovered after traveling (Table 2 ).

\section{Antibiotic Susceptibility of ESC-R-Ent}

As shown in Table 3, none of the isolates detected in the post-travel stools were resistant to piperacillin/tazobactam or carbapenems. For non- $\beta$-lactams, higher rates of resistance $(\sim 35-40 \%)$ were observed for doxycycline, levofloxacin, ciprofloxacin, trimethoprim-sulfamethoxazole (STX), whereas resistance to minocycline and aminoglycosides was $\leq 20 \%$ and none of the isolates was resistant to tigecycline.

During the follow up periods, the prevalence of resistance to fluoroquinolones and to minocycline increased, whereas for STX and aminoglycosides the resistance rates continuously decreased (Table 3).

\section{$\beta$-lactamase Diversity over Time}

Pre-travel, the E. coli strains identified were CTX-M-15- and CTX-M-14 producers (Table 2). Of the 28 isolates recovered after traveling (26 E. coli, one K. pneumoniae, and one P. mirabilis), $89.3 \%$ were ESBL producers and the remaining $10.7 \%$ were pAmpC producers. CTX-M-15 was the most frequent ESBL identified (92.0\%), followed by SHV-12 and VEB-6 (both 4\%).

In the follow up period, a decreased in the diversity was observed. At 3 months, 75\% of the strains produced CTX-M15 , while CTX-M-14 and CMY-42 were produced by $12.5 \%$ each. At 6 months, strains produced CTX-M-15 (42.9\%), CTX-M1(28.6\%), CTX-M-24-like, and CMY-42 (14.3\% each).

\section{Population Structure of ESC-R-Ent}

E. coli strains were highly diverse, with clones belonging to different STs and PhGs (Table 2). Before traveling, B2 $2_{3}-\mathrm{ST} 131$, $\mathrm{D}_{2}$-ST648, and $\mathrm{A}_{1}$-ST652 strains were detected.

Among the $26 \mathrm{E}$. coli identified after returning from the trip, $22(88 \%)$ yielded an individual ST. Nevertheless, 4 isolates (15\%) belonged to the clonal complex (CC) 10. The PhG A was predominant (50\%), followed by B1 and D (27 and 19\%, respectively), being $\mathrm{B} 2 \mathrm{PhG}$ almost absent.

At 3 months, B2 or B1 PhGs were not identified, whereas PhGs $\mathrm{A}$ and $\mathrm{D}$ were equally represented. At 6 months, PhG D was the most frequent (57\%), followed by A (29\%) and B1 (14\%) strains.
With regard to plasmids, those included in the F family were the most frequently identified $(\sim 80 \%)$, even though few other replicon types were observed (i.e., $\mathrm{X} 1, \mathrm{I} 1, \mathrm{~B} / \mathrm{O}$, and $\mathrm{K}$ ). F plasmids were also seen more frequently at 3 and 6 months, whereas other types identified after traveling were no longer recorded (Table 2).

\section{Shifts in Molecular Characteristics Over Time}

Shifts in the molecular characteristics of the ESC-R-Ent recovered at the different time points were observed (Table 2). Three months after traveling, six HVs (HVs \#26, 34, 43, 59, 97, and 100) were decolonized regardless of the number of clones identified immediately after return. For three HVs not colonized at 3 months (HVs \#23, 37, 41), ESC-R-Ent were identified at 6 months; compared to each other, these strains carried a different $\beta$-lactamase type in a different ST and had a

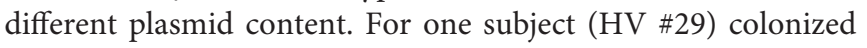
before traveling, the clone recovered just after the trip was different than the initial one; however, 3 months later the initial clone $\left(\mathrm{D}_{2}\right.$-ST648-CTX-M-14) reappeared again (Supplemental Figure 1).

Persistent clones were also observed in HVs \#56 and \#80 as the same ST, plasmid content, and $\beta$-lactamase were identified at 3 and 6 months, respectively (Supplemental Figure 1). In the case of $\mathrm{HV} \# 83$, there was a shift of clones and $\beta$ lactamases from the stools collected after traveling to those collected at 3 months; the same clone $\left(\mathrm{D}_{2}\right.$-ST648) was identified after 6 months. Persistence of the $\beta$-lactamase in the same mobile genetic element (MGE) was suspected in the case of $\mathrm{HV} \# 50$, since the same replicon types identified in the ST155 strain detected after traveling was identified in the ST394 detected at 3 and 6 months (Table 2, Supplemental Figure 1).

\section{Carriage of Multiple ESC-R-Ent}

After returning from India, 9 (60\%) subjects carried more than one ESC-R-Ent, with a mean of 1.9 clones and a median of 2 clones (overall range of 1-5). Different E. coli STs were frequently identified colonizing the same individual harboring the same ESBL type. Nonetheless, carriage of different $\beta$-lactamases was also observed (HVs \#34 and \#43).

At 3 and 6 months, carriage of multiple clones was identified only in $\mathrm{HV} \# 68$ and $\mathrm{HV} \# 37$, respectively. In particular, $\mathrm{HV} \# 68$ had three different CTX-M-15 E. coli producers at 3 months, whereas HV \#37 had at 6 months two different STs harboring dissimilar CTX-M-types.

When different species were found together with E. coli, they could carry the same bla (i.e., CTX-M-15-producing $K$. pneumoniae for HV \#26), or a different gene (i.e., VEB-6producing $P$. mirabilis for $\mathrm{HV} \# 37$ ).

\section{DISCUSSION}

In this study, we prospectively followed a group of $15 \mathrm{HVs}$ who returned from India colonized at intestinal level with more than one ESC-R-Ent clone. We emphasize that only a few studies have explored the issue of the polyclonal colonization of travelers with 


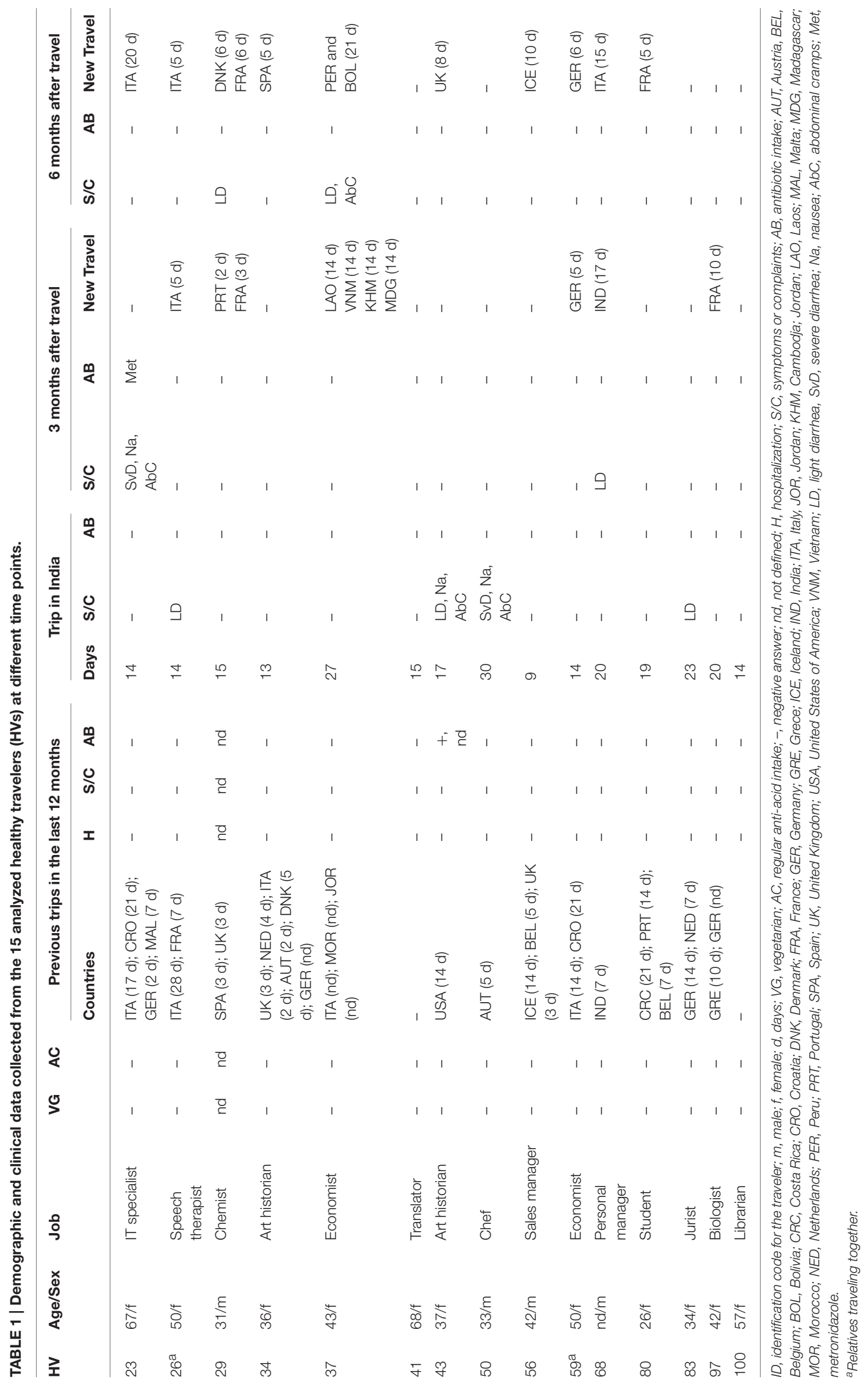




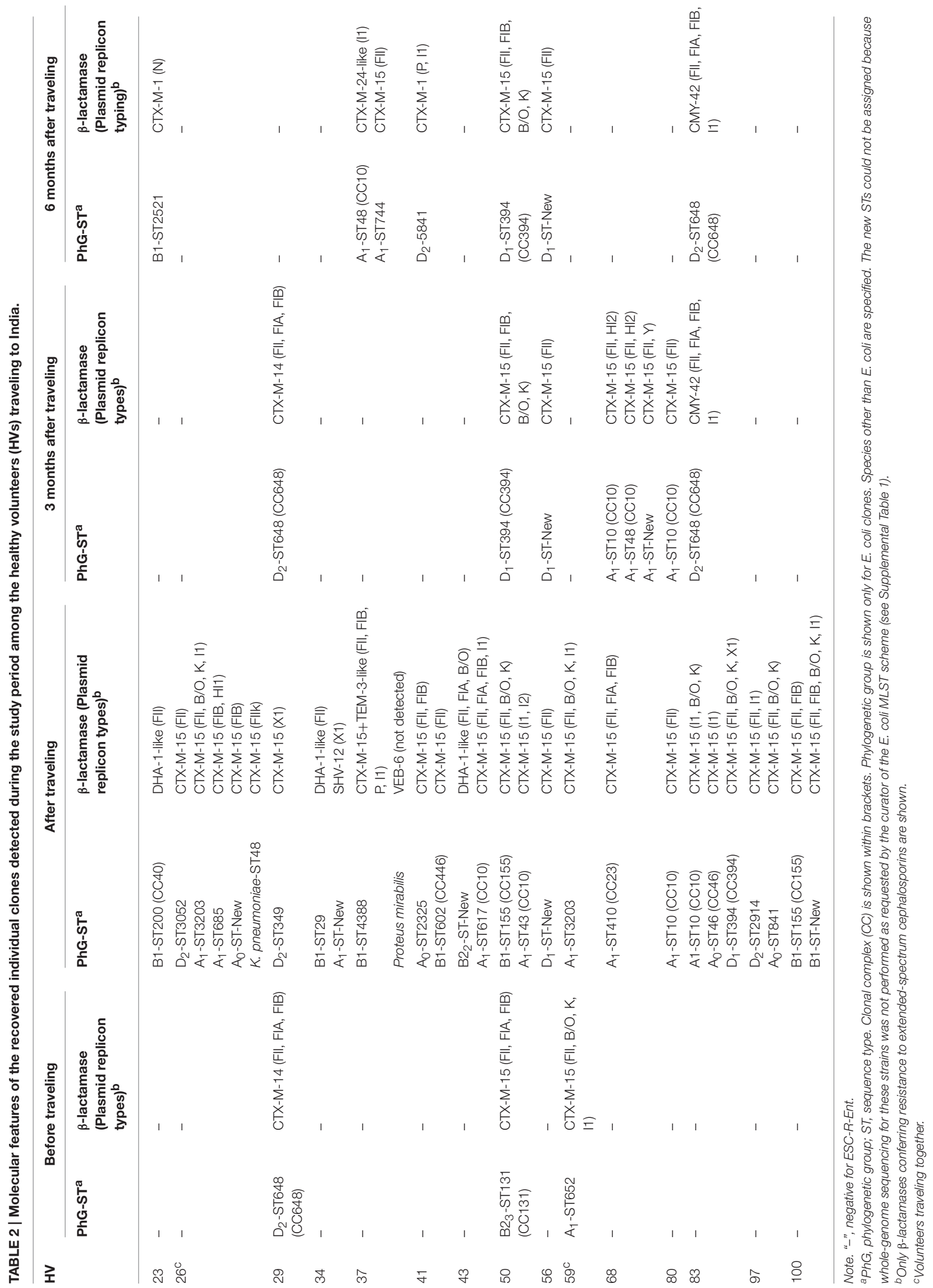


TABLE 3 | Distribution of $\beta$-lactamase types, antibiotic resistance rates and E. coli phylogenetic groups for the 15 volunteers.

$$
\begin{array}{ccccc}
\begin{array}{c}
\text { Overall period } \\
\left(n=46, n^{\circ}\right. \text { isolates, \%) }
\end{array} & \begin{array}{c}
\text { Before Traveling } \\
\left(n=3 n^{\circ}\right. \text { isolates, \%) }
\end{array} & \begin{array}{c}
\text { After Traveling } \\
\left(n=28, n^{\circ}\right. \text { isolates, \%) }
\end{array} & \begin{array}{c}
3 \text { months after } \\
\text { traveling } \\
\left(n=8, n^{\circ}\right. \text { isolates, \%) }
\end{array} & \begin{array}{c}
6 \text { months after } \\
\text { traveling }
\end{array} \\
& & & \left(n=7, n^{\circ}\right. \text { isolates, \%) }
\end{array}
$$

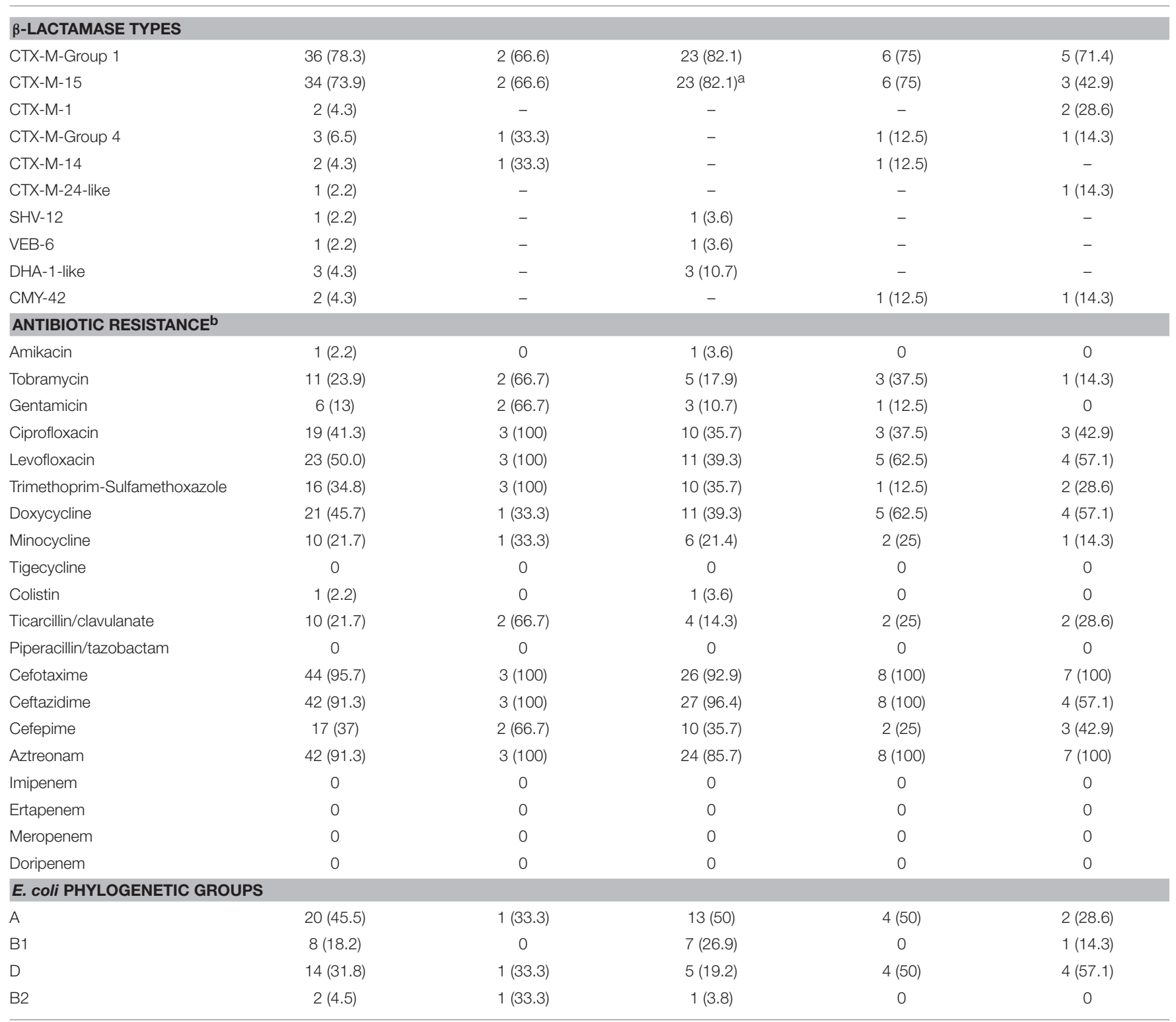

a One isolates co-carries a TEM-3-like.

${ }^{b}$ Based on EUCAST breakpoints except for doxycycline and minocycline for which CLSI was used.

these life-threatening Gram-negative pathogens (Paltansing et al., 2013; Girlich et al., 2015; Stoesser et al., 2015).

\section{Antibiotic Susceptibility and $\beta$-lactamase Diversity}

The resistance profiles of the recovered ESC-R-Ent isolates (i.e., higher rates of resistance to fluoroquinolones, STX, and aminoglycosides) clearly emphasized the limited option available for antibiotic therapy in case of infection due to these pathogens.
As expected, the most prevalent $\beta$-lactamase types identified in this study were the CTX-M-15 (Kennedy and Collignon, 2010; Tängdén et al., 2010; Peirano et al., 2011; Östholm-Balkhed et al., 2013; Paltansing et al., 2013; Kuenzli et al., 2014; Sole et al., 2014; Lübbert et al., 2015; Ruppé et al., 2015; Valverde et al., 2015; Barreto Miranda et al., 2016), whereas other $\beta$ lactamases (i.e., SHV-12-like, CMY-2-like, and DHA-type) were rarely detected (Östholm-Balkhed et al., 2013; Kuenzli et al., 2014; Sole et al., 2014). The frequent observation of F plasmids was also consistent with the great dispersion of these MGEs 
carrying bla $\mathrm{CTX}-\mathrm{M}$-types among Enterobacteriaceae recovered from different settings worldwide (Carattoli, 2013; Mathers et al., 2015).

\section{Population Structure Shifts among ESC-R-Ent in Returning Travelers}

Prior to travel, the HiRC B23-ST131 and $\mathrm{D}_{2}-\mathrm{ST} 648$ were identified. These PhGs/STs have been found worldwide colonizing and/or causing infections in humans, both companion and food-producing animals, and wildlife (Woodford et al., 2011; Ewers et al., 2012, 2014; Pitout, 2012; Nakane et al., 2016). Interestingly, in the HVs analyzed these initial clones were replaced after the trip to India (Table 2).

Post-travel, the high heterogeneity of clones identified indicated numerous possible exposure sources with ESC-R-Ent. Given that isolates belonged mostly to $\mathrm{A}$ and $\mathrm{B} 1 \mathrm{PhGs}$ and to $\mathrm{CC} 10, \mathrm{CC} 155$, or CC23, we speculate that the acquisition of these clones could be linked to environmental exposure and/or the food-chain (Paltansing et al., 2013; Kuenzli et al., 2014; Valverde et al., 2015). Moreover, the fact that we found ESBL-Ent coding for the same $\beta$-lactamase enzymes, which are frequently reported in food-items and river waters, strengthens this hypothesis (Bajaj et al., 2015; Upadhyay et al., 2015; Zurfluh et al., 2015).

However, we should note that despite being unlikely, humanto-human transmission cannot be excluded, since people living in tropical or sub-tropical climates have PhG A E. coli strains as the predominant lineages in the gut (Escobar-Páramo et al., 2004; Tenaillon et al., 2010). One Indian study identified CTXM-15-producing E. coli of PhG A as the most frequent ESBL-E. coli lineage colonizing the gut of healthy individuals (EscobarPáramo et al., 2004; Dureja et al., 2014). Furthermore, the identification of $\mathrm{A}_{1}$-ST3203 in two HVs traveling together (\#26 and \#59), but having different pre-travel colonization status (Table 2), may suggest that the acquisition arises from a common exposure source or human-to-human transmission. Overall, the increasing recovery of PhG A and B1 E. coli is worrisome, due to their expansion in the clinical setting associated with the production of ESBLs (Woodford et al., 2011; Rodrigues et al., 2015).

In the stools collected at 3 and 6 months, several epidemiological shifts were observed. The persistence of the same clone was observed until 3 months for HV \#80 and until 6 months for HV \#56. Nevertheless, the identification of the same replicon types in a different ST, as observed for HV $\# 50$, suggests that in vivo conjugation may occur (van Schaik, 2015). However, we cannot exclude that the different clones identified during the follow-up can also be associated to new independent acquisitions, as several of these HVs have traveled to high prevalence countries during this period (HVs \#23, 37, and 68; Table 1).

Among the different STs that could be consistently identified over time, ST648 and members of CC10 were the most frequent (Table 2). In addition, the majority of plasmids recovered belonged to the $\mathrm{F}$ family. This combination of clones and plasmids, which can easily adapt to the human host, could explain their persistence in the gut (Paltansing et al., 2013; Titelman et al., 2014). Intrinsic characteristics of these genetic backgrounds or factors conferring increased fitness (e.g., usage of alternative metabolic pathways), might also contribute to the persistent intestinal colonization (Alteri and Mobley, 2012).

\section{Colonization Dynamics: Hypothetical Pathways}

According to our observations spanning a 6-month period, several pathways can be drawn when an initially non-colonized HV travels to a high prevalence setting: (i) clonal persistence after returning from the trip (e.g., HV \#56); (ii) persistence of the MGE (e.g., HV \#50); (iii) detection of a previously unidentified clone or independent new acquisition (e.g., HV \#83), and (iv) clonal clearance (e.g., HV \#26) from the intestinal tract after several months (Table 2).

In the case of previously colonized HVs, an additional pathway can be identified, as the initial clone can be still present after traveling, but below the detection limit and then re-emerges after some time (e.g., HV \#29) (Paltansing et al., 2013). This phenomenon has been observed in patients colonized after an infection (Titelman et al., 2014).

\section{Polyclonality: Clinical and Ecological Implications}

In our study, a median of 2 clones were identified in the stools of travelers after the trip, indicating that a polyclonal acquisition occurs when visiting a high ESC-R-Ent prevalence area. Interestingly, HV \#68 returned to India in the follow-up period and then he was found colonized with three unique MDR clones, emphasizing the risk of polyclonal acquisition in this region. This overall phenomenon has been already perceived by other authors (Östholm-Balkhed et al., 2013; Paltansing et al., 2013; Lübbert et al., 2015; Ruppé et al., 2015; Valverde et al., 2015). However, only one study reported a mean of 1.8 clones among the travelers (being the polyclonal acquisition identified in $46 \%$ of them), but no bacterial population structure analysis was performed (Ruppé et al., 2015).

In our opinion, there are several important implications associated with the polyclonal acquisition of ESC-R-Ent. On the clinical level, physicians should be aware that extra-intestinal infections in patients who recently traveled in high ESC-REnt prevalence region might be due to MDR clones that are persisting in the gut. Therefore, the antibiotic treatment has to be tailored according to the ASTs results provided by the clinical laboratory and not empirically given (Kennedy and Collignon, 2010). At the diagnostic laboratory level, colony picking from the selective agar plates can lead to the identification of just one MDR isolate (e.g., identifying an ESBL-Ent ciprofloxacin susceptible, but not the ESBL-Ent resistant to the antibiotic), hindering the implementation of proper antibiotic treatment regiments (Kennedy and Collignon, 2010; Endimiani and Jacobs, 2016). At the colonization level, different pathways are possible. The genetic background of the bacterium could have a significant influence on its ability to persist in the gut. Moreover, in vivo conjugation can also propagate resistance mechanisms and/or novel adaptive characteristics into new bacterial hosts with 
stronger ability to persistently colonize the human gut (e.g., the HiRCs ST131 and ST648), further potentiating the overall spread of these MDR pathogens (Baquero et al., 2011; van Schaik, 2015). Moreover, it has also been demonstrated that bacteria with certain genetic backgrounds (ST131, ST648, and those of PhG A) do not suffer fitness upon acquisition of MDR plasmids. Therefore, particular combinations of very fitting STs with MDR plasmids might favor more successful colonization overtime (Johnson et al., 2015; Schaufler et al., 2016).

\section{CONCLUSIONS}

A high polyclonal acquisition rate of ESC-R-Ent was observed when HVs traveled to India. These multiple clones can follow different pathways, ranging from clonal clearance to clonal and/or MGE persistence in the gut. The genetic backgrounds of the acquired clones suggest that this acquisition is linked to not yet well-defined non-human sources, with the food-chain and the environment being likely potential sources.

The effective implications of these overall findings are still not completely clear. However, it appears plausible that travel medicine practitioners, clinicians, and clinical microbiologists who are facing the returning travelers and their specimens for different clinical reasons should be aware of this important phenomenon, so that better infection control measures, treatment strategies, and diagnostic tests can be adopted. Studying the factors underlying ESC-R-Ent persistence in the gut is also extremely important to better devise

\section{REFERENCES}

Alteri, C. J., and Mobley, H. L. T. (2012). Escherichia coli physiology and metabolism dictates adaptation to diverse host microenvironments. Curr. Opin. Microbiol. 15, 3-9. doi: 10.1016/j.mib.2011.12.004

Bajaj, P., Singh, N. S., Kanaujia, P. K., and Virdi, J. S. (2015). Distribution and molecular characterization of genes encoding CTX-M and AmpC $\beta$-lactamases in Escherichia coli isolated from an Indian urban aquatic environment. Sci. Total Environ. 505, 350-356. doi: 10.1016/j.scitotenv.2014.09.084

Baquero, F., Coque, T. M., and De La Cruz, F. (2011). Ecology and evolution as targets: the need for novel eco-evo drugs and strategies to fight antibiotic resistance. Antimicrob. Agents Chemother. 55, 3649-3660. doi: 10.1128/AAC.00013-11

Barreto Miranda, I., Ignatius, R., Pfüller, R., Friedrich-Jänicke, B., Steiner, F., Paland, M., et al. (2016). High carriage rate of ESBL-producing Enterobacteriaceae at presentation and follow-up among travellers with gastrointestinal complaints returning from India and Southeast Asia. J. Travel Med. 23:tav024. doi: 10.1093/jtm/tav024

Bernasconi, O. J., Kuenzli, E., Pires, J., Tinguely, R., Carattoli, A., Hatz, C., et al. (2016). Travelers can import colistin-resistant enterobacteriaceae including those possessing the plasmid-mediated mcr-1 gene. Antimicrob. Agents Chemother. doi: 10.1128/AAC.00731-16. [Epub ahead of print].

Carattoli, A. (2013). Plasmids and the spread of resistance. Int. J. Med. Microbiol. 303, 298-304. doi: 10.1016/j.ijmm.2013.02.001

CLSI (2016). Performances Standards for Antimicrobial Susceptibility Testing; Twenty-Fourth Informational Supplement. M100-S26. Wayne, PA: Clinical Laboratory Standards Institute.

Dureja, C., Mahajan, S., and Raychaudhuri, S. (2014). Phylogenetic distribution and prevalence of genes encoding class I integrons and CTX-M-15 extendedspectrum $\beta$-lactamases in Escherichia coli isolates from healthy humans in Chandigarh, India. PLoS ONE 9:e112551. doi: 10.1371/journal.pone.0112551 decolonization and colonization prevention strategies in the near future.

\section{AUTHOR CONTRIBUTIONS}

Conception and design (EK, CH, AE); acquisition of data (JP, EK, SK, RT); analysis and interpretation of data (JP, EK, HF, MH, CH, $\mathrm{AE}$ ); drafting the work (JP, AE); revising it critically for important intellectual content (all authors); final approval of the version to be published (all authors); agreement to be accountable for all aspects of the work in ensuring that questions related to the accuracy or integrity of any part of the work are appropriately investigated and resolved (all authors).

\section{FUNDING}

This work was supported in part by the Swiss National Science Foundation (SNF; grant number 153377 to AE).

\section{ACKNOWLEDGMENTS}

JP is a PhD student (2014-2017) supported by the SNF.

\section{SUPPLEMENTARY MATERIAL}

The Supplementary Material for this article can be found online at: http://journal.frontiersin.org/article/10.3389/fmicb. 2016.01069
Endimiani, A., and Jacobs, M. R. (2016). The changing role of the clinical microbiology laboratory in defining resistance in gram-negatives. Infect. Dis. Clin. North Am. 30, 323-345. doi: 10.1016/j.idc.2016.02.002

Escobar-Páramo, P., Grenet, K., Le Menac'H, A., Rode, L., Salgado, E., Amorin, C., et al. (2004). Large-scale population structure of human commensal Escherichia coli isolates. Appl. Environ. Microbiol. 70, 5698-5700. doi: 10.1128/AEM.70.9.5698-5700.2004

Eucast (2016). European Committee on Antimicrobial Susceptibility Testing. Breakpoint tables for interpretation of MICs and zone diameters Version 6.0. Available online at: www.eucast.org

Ewers, C., Bethe, A., Semmler, T., Guenther, S., and Wieler, L. H. (2012). Extendedspectrum $\beta$-lactamase-producing and AmpC-producing Escherichia coli from livestock and companion animals, and their putative impact on public health: a global perspective. Clin. Microbiol. Infect. 18, 646-655. doi: 10.1111/j.14690691.2012.03850.x

Ewers, C., Bethe, A., Stamm, I., Grobbel, M., Kopp, P. A., Guerra, B., et al. (2014). CTX-M-15-D-ST648 Escherichia coli from companion animals and horses: another pandemic clone combining multiresistance and extraintestinal virulence? J. Antimicrob. Chemother. 69, 1224-1230. doi: 10.1093/jac/d kt516

Girlich, D., Nordmann, P., Lécuyer, H., Berche, P., Marmorat-Khuong, A., Gros, I., et al. (2015). Multiple colonization with highly resistant bacteria: carbapenemase-producing Enterobacteriaceae, carbapenemase-producing Pseudomonas aeruginosa, carbapenemase-producing Acinetobacter baumannii, and glycopeptide-resistant Enterococcus faecium. Diagn. Microbiol. Infect. Dis. 81, 217-218. doi: 10.1016/j.diagmicrobio.2014.12.002

Johnson, T. J., Singer, R. S., Isaacson, R. E., Danzeisen, J. L., Lang, K., Kobluk, $\mathrm{K}$., et al. (2015). In vivo transmission of an IncA/C Plasmid in escherichia coli depends on tetracycline concentration, and acquisition of the plasmid results in a variable cost of fitness. Appl. Environ. Microbiol. 81, 3561-3570. doi: 10.1128/AEM.04193-14 
Kennedy, K., and Collignon, P. (2010). Colonisation with Escherichia coli resistant to "critically important" antibiotics: a high risk for international travellers. Eur. J. Clin. Microbiol. Infect. Dis. 29, 1501-1506. doi: 10.1007/s10096-010-1031-y

Kuenzli, E., Jaeger, V., Frei, R., Neumayr, A., Decrom, S., Haller, S., et al. (2014). High colonization rates of extended-spectrum beta-lactamase (ESBL)producing Escherichia coli in Swiss Travellers to South Asia- a prospective observational multicentre cohort study looking at epidemiology, microbiology and risk factors. BMC Infect. Dis. 14:528. doi: 10.1186/1471-2334-14-528

Lübbert, C., Straube, L., Stein, C., Makarewicz, O., Schubert, S., Mössner, J., et al. (2015). Colonization with extended-spectrum beta-lactamaseproducing and carbapenemase-producing Enterobacteriaceae in international travelers returning to Germany. Int. J. Med. Microbiol. 305, 148-156. doi: 10.1016/j.ijmm.2014.12.001

Mathers, A. J., Peirano, G., and Pitout, J. D. D. (2015). The role of epidemic resistance plasmids and international high-risk clones in the spread of multidrug-resistant Enterobacteriaceae. Clin. Microbiol. Rev. 28, 565-591. doi: 10.1128/CMR.00116-14

Memish, Z. A., Venkatesh, S., and Shibl, A. M. (2003). Impact of travel on international spread of antimicrobial resistance. Int. J. Antimicrob. Agents 21, 135-142. doi: 10.1016/S0924-8579(02)00363-1

Nakane, K., Kawamura, K., Goto, K., and Arakawa, Y. (2016). Long-term colonization by $b l a_{C T X}-M$-harboring Escherichia coli in healthy japanese people engaged in food handling. Appl. Environ. Microbiol. 82, 1818-1827. doi: 10.1128/AEM.02929-15

Östholm-Balkhed, Å., Tärnberg, M., Nilsson, M., Nilsson, L. E., Hanberger, H., Hällgren, A., et al. (2013). Travel-associated faecal colonization with ESBL-producing Enterobacteriaceae: incidence and risk factors. J. Antimicrob. Chemother. 68, 2144-2153. doi: 10.1093/jac/dkt167

Paltansing, S., Vlot, J. A., Kraakman, M. E. M., Mesman, R., Bruijning, M. L., Bernards, A. T., et al. (2013). Extended-spectrum $\beta$-lactamase-producing Enterobacteriaceae among travelers from the Netherlands. Emerg. Infect. Dis. J. 19, 1206. doi: 10.3201/eid1908.130257

Peirano, G., Laupland, K. B., Gregson, D. B., and Pitout, J. D. D. (2011). Colonization of returning travelers with CTX-M-producing Escherichia coli. J. Travel Med. 18, 299-303. doi: 10.1111/j.1708-8305.2011.00548.x

Pitout, J. D. D. (2012). Extraintestinal pathogenic Escherichia coli: a combination of virulence with antibiotic resistance. Front. Microbiol. 3:9. doi: 10.3389/fmicb.2012.00009

Rodrigues, C., Machado, E., Pires, J., Ramos, H., Novais, Â., and Peixe, L. (2015). Increase of widespread A, B1 and D Escherichia coli clones producing a high diversity of CTX-M-types in a Portuguese hospital. Future Microbiol. 10, 1125-1131. doi: 10.2217/fmb.15.38

Rogers, B. A., Aminzadeh, Z., Hayashi, Y., and Paterson, D. L. (2011). Country-tocountry transfer of patients and the risk of multi-resistant bacterial infection. Clin. Infect. Dis. 53, 49-56. doi: 10.1093/cid/cir273

Ruppé, E., Armand-Lefèvre, L., Estellat, C., Consigny, P.-H., El Mniai, A., Boussadia, Y., et al. (2015). High rate of acquisition but short duration of carriage of multidrug-resistant Enterobacteriaceae after travel to the tropics. Clin. Infect. Dis. 61, 593-600. doi: 10.1093/cid/civ333

Schaufler, K., Semmler, T., Pickard, D. J., De Toro, M., De La Cruz, F., Wieler, L. H., et al. (2016). Carriage of extended-spectrum beta-lactamase-plasmids does not reduce fitness but enhances virulence in some strains of pandemic E. coli lineages. Front. Microbiol. 7:336. doi: 10.3389/fmicb.2016.00336

Seiffert, S. N., Hilty, M., Kronenberg, A., Droz, S., Perreten, V., and Endimiani, A. (2013). Extended-spectrum cephalosporin-resistant Escherichia coli in community, specialized outpatient clinic and hospital settings in Switzerland. J. Antimicrob. Chemother. 68, 2249-2254. doi: 10.1093/jac/dkt208

Seiffert, S. N., Perreten, V., Johannes, S., Droz, S., Bodmer, T., and Endimiani, A. (2014). OXA-48 carbapenemase-producing Salmonella enterica serovar kentucky isolate of sequence type 198 in a patient transferred from Libya to Switzerland. Antimicrob. Agents Chemother. 58, 2446-2449. doi: 10.1128/AAC.02417-13

Short, F. L., Murdoch, S. L., and Ryan, R. P. (2014). Polybacterial human disease: the ills of social networking. Trends Microbiol. 22, 508-516. doi: 10.1016/j.tim.2014.05.007

Sole, M., Pitart, C., Oliveira, I., Fábrega, A., Muñoz, L., Campo, I., et al. (2014). Extended spectrum $\beta$-lactamase-producing Escherichia coli faecal carriage in
Spanish travellers returning from tropical and subtropical countries. Clin. Microbiol. Infect. 20, O636-O639. doi: 10.1111/1469-0691.12592

Stoesser, N., Xayaheuang, S., Vongsouvath, M., Phommasone, K., Elliott, I., Del Ojo Elias, C., et al. (2015). Colonization with Enterobacteriaceae producing ESBLs in children attending pre-school childcare facilities in the Lao People's Democratic Republic. J. Antimicrob. Chemother. 70, 1893-1897. doi: 10.1093/jac/dkv021

Tängdén, T., Cars, O., Melhus, Å., and Löwdin, E. (2010). Foreign travel is a major risk factor for colonization with Escherichia coli producing CTX-M-type extended-spectrum $\beta$-lactamases: a prospective study with swedish volunteers. Antimicrob. Agents Chemother. 54, 3564-3568. doi: 10.1128/AAC.00220-10

Tenaillon, O., Skurnik, D., Picard, B., and Denamur, E. (2010). The population genetics of commensal Escherichia coli. Nat. Rev. Microbiol. 8, 207-217. doi: 10.1038/nrmicro2298

Titelman, E., Hasan, C. M., Iversen, A., Nauclér, P., Kais, M., Kalin, M., et al. (2014). Faecal carriage of extended-spectrum $\beta$-lactamase-producing Enterobacteriaceae is common 12 months after infection and is related to strain factors. Clin. Microbiol. Infect. 20, O508-O515. doi: 10.1111/1469-0691. 12559

Upadhyay, S., Hussain, A., Mishra, S., Maurya, A. P., Bhattacharjee, A., and Joshi, S. R. (2015). Genetic environment of plasmid mediated CTX-M-15 extended spectrum beta-lactamases from clinical and food borne bacteria in NorthEastern India. PLoS ONE 10:e0138056. doi: 10.1371/journal.pone.0138056

Valverde, A., Turrientes, M. C., Norman, F., San Martín, E., Moreno, L., PérezMolina, J. A., et al. (2015). CTX-M-15-non-ST131 Escherichia coli isolates are mainly responsible of faecal carriage with ESBL-producing Enterobacteriaceae in travellers, immigrants and those visiting friends and relatives. Clin. Microbiol. Infect. 21, 252.e1-4. doi: 10.1016/j.cmi.2014.09.021

Van Der Bij, A. K., and Pitout, J. D. D. (2012). The role of international travel in the worldwide spread of multiresistant Enterobacteriaceae. J. Antimicrob. Chemother. 67, 2090-2100. doi: 10.1093/jac/dks214

van Schaik, W. (2015). The human gut resistome. Philoso. Trans. R. Soc. B Biol. Sci. 370:20140087. doi: 10.1098/rstb.2014.0087

Viau, R., Frank, K. M., Jacobs, M. R., Wilson, B., Kaye, K., Donskey, C. J., et al. (2016). Intestinal carriage of carbapenemase-producing organisms: current status of surveillance methods. Clin. Microbiol. Rev. 29, 1-27. doi: 10.1128/CMR.00108-14

Woerther, P.-L., Burdet, C., Chachaty, E., and Andremont, A. (2013). Trends in human fecal carriage of extended-spectrum $\beta$-lactamases in the community: toward the globalization of CTX-M. Clin. Microbiol. Rev. 26, 744-758. doi: 10.1128/CMR.00023-13

Woodford, N., Turton, J. F., and Livermore, D. M. (2011). Multiresistant Gram-negative bacteria: the role of high-risk clones in the dissemination of antibiotic resistance. FEMS Microbiol. Rev. 35, 736-755. doi: 10.1111/j.15746976.2011.00268.x

Yong, D., Toleman, M. A., Giske, C. G., Cho, H. S., Sundman, K., Lee, K., et al. (2009). Characterization of a new metallo- $\beta$-lactamase gene, blaNDM- 1 , and a novel erythromycin esterase gene carried on a unique genetic structure in Klebsiella pneumoniae sequence type 14 from India. Antimicrob. Agents Chemother. 53, 5046-5054. doi: 10.1128/AAC.00774-09

Zurfluh, K., Nüesch-Inderbinen, M., Morach, M., Zihler Berner, A., Hächler, H., and Stephan, R. (2015). Extended-spectrum- $\beta$-lactamase-producing Enterobacteriaceae isolated from vegetables imported from the dominican republic, India, Thailand, and Vietnam. Appl. Environ. Microbiol. 81, 3115-3120. doi: 10.1128/AEM.00258-15

Conflict of Interest Statement: The authors declare that the research was conducted in the absence of any commercial or financial relationships that could be construed as a potential conflict of interest.

Copyright (ㅇ 2016 Pires, Kuenzli, Kasraian, Tinguely, Furrer, Hilty, Hatz and Endimiani. This is an open-access article distributed under the terms of the Creative Commons Attribution License (CC BY). The use, distribution or reproduction in other forums is permitted, provided the original author(s) or licensor are credited and that the original publication in this journal is cited, in accordance with accepted academic practice. No use, distribution or reproduction is permitted which does not comply with these terms. 